\title{
Correction to "Design, Synthesis, and Antibacterial Evaluation of Oxazolidinones with Fused Heterocyclic C-Ring Substructure"
}

Mahesh S. Deshmukh and Nidhi Jain*(1)

ACS Med. Chem. Lett. 2017, 8 (11), pp 1153-1158. DOI: 10.1021/acsmedchemlett.7b00263

Supporting Information

$\mathrm{O}$ ur previously published article "Design, Synthesis, and Antibacterial Evaluation of Oxazolidinones with Fused Heterocyclic C-Ring Substructure" (DOI: 10.1021/acsmedchemlett.7b00263) was published with an incorrect Supporting Information file. The correct Supporting Information file can be found here.

\section{ASSOCIATED CONTENT}

\section{S Supporting Information}

The Supporting Information is available free of charge on the ACS Publications website at DOI: 10.1021/acsmedchemlett.8b00164.

Experimental procedures, compound characterization details, protocols for biological evaluation, and molecular docking studies (PDF) 